\title{
Einige seltene angeborene Anomalien des Auges.
}

\author{
Von \\ Prof. Eugen v. Hippel \\ in Heidelberg.
}

Mit 2 Figuren im Text.

\section{Anatomische Untersuchung \\ eines mit partiellem angeborenem Irismangel nach oben innen und Hämophthalmus behafteten Auges.}

Das fünf Monate alte Söhnchen eines Arztes wurde am 20. VII. 1900 in die Augenklinik zu Heidelberg aufgenommen wegen einer Erkrankung des linken Auges, die von augenärztlicher Seite mit Wahrscheinlichkeit als Gliom diagnosticirt worden war.

Die Anamnese ergab Folgendes: Die zwei Tage lang danernde Geburt des Kindes musste mit der Zange beendigt werden. Der mir persönlich befreundete behandelnde Gynäkologe hatte keine Anomalie an den Augen des Kindes bemerkt, wie aber kaum zu bezweifeln ist, weil er, durch die Sorge für die Mutter in Anspruch genommen, nicht daranf geachtet hatte. Der Vater des Kindes hatte gleich am ersten Tage bemerkt, dass das linke Auge nicht in Ordnnng war, dasselbe zeigte eine subconjunctivale Blutung, ausserdem war die ganze vordere Kammer mit Blut erfüllt. Die Pupille wurde erst nach einigen Tagen sichtbar und ${ }_{3}$ war vollständig verzerrt", doch ist nicht vollkommene Sicherheit darüber zu erlangen, ob die Anomalie der Pupille bereits in den ersten Lebenstagen die gleiche gewesen ist, wie zu der Zeit, als das Kind ron uns untersucht wurde. Als das Kind drei Wochen alt war, wurde ein gelbrother Reflex aus der Tiefe, besonders oben innen, in den folgenden Wochen ein geringes Zurüekbleiben des Auges im Wachsthum bemerkt. Der Reflex aus der Tiefe nahm allmählich eine gelbgraue Farbe an. Im dritten oder vierten Lebensmonat entzündete sich das Auge und wurde sehmerahaft.

Status: (zum Theil erst in Narkose ganz genau zu erheben).

R. A. in jeder Hinsicht normal.

L. A. etwas kleiner als rechtes. Conjunctiva etwas durch Blutfarbstoff verfärbt, Cornea klar, vordere Kammer tief. Iris atrophisch, Pupille reactionslos, weit und von ganz unregelmässiger Form: Oben innen und oben ist überhaupt keine Iris $z u$ sehen, unten aussen ist die Iris dagegen am breitesten. Entsprechend der Stelle des Irisdefectes sieht man die v. Graefe's Archiv für Ophthalmologie. LII. 3. 
weit vorgezerrten Ciliarfortsätze mit grosser Deutlichkeit. Vor lenselben sieht man eine kleine Blutung, die sich nach linten in den Glaskörper erstreekt. Linse klar. Hinter derselben ein graugrines Exsudat, keine Gefässe auf demselben zu sehen.

Wahrscheinlichkeitsdiagnose: Ausgänge hochgradiger intraocularer Blutungen; da indessen Gliom nicht sicher auszuschliessen und das Auge blind war, wuxde die Enucleation ansgefilmt. Dabei wird eine anffallende Härte des enudeinten Bulbus bemerkt.

Anteropost. Durchmesser 20, horizontaler und verticaler $19^{1 / 2} \mathrm{~mm}$. Die Cornea ist auffallend stark gewolbt. Oben innen in der Richtung des Irisdefectes ist die Sklerocomealgrenze eingezogen. Dies beruht, wie die mikroskopische Untersuchung ergiebt, auf einem an dieser Stelle besonders stark von innen her wirkenden Zuge.

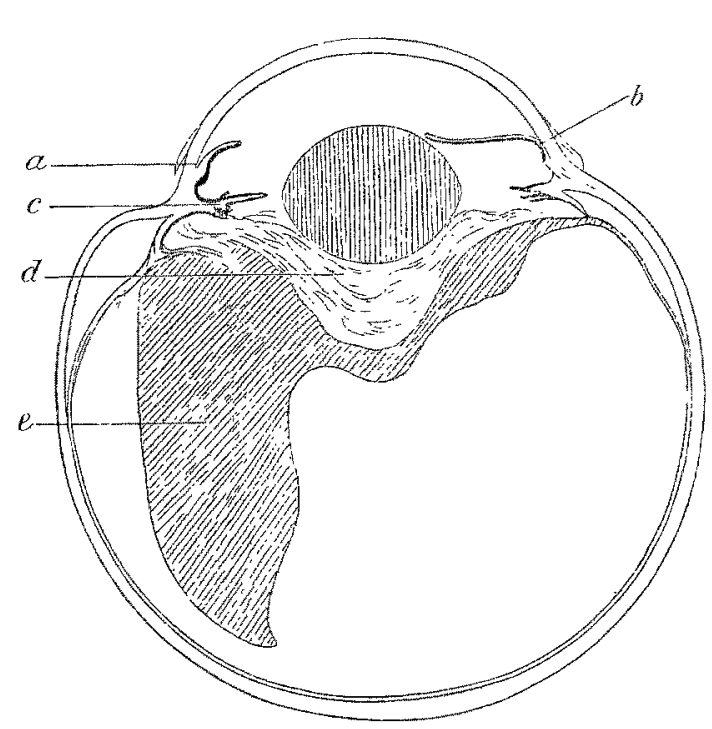

Fig. 1.

Härtung in Zenkerseher Lösung, Serienschnitte in der Richtung von oben innen nach unten aussen, entsprechend dem Irisdefect.

Die makroskopische Betrachtung der Schnitte lässt eine totale Netzhantablösung und eine grosse Blutung in subretinalen Raum erkennen. Mikroskopischer Befund: Die Cornea zeigt bistologisel keine Veränderung, ist aber etwas vergrössert und abnorm stark gewölbt. Die Iriswurzel ist überall mit der Hornhauthinterfläche fest verwachsen, so dass der Filtrationswinkel gesperrt ist. Der Schlemm'sche Plexus ist vorhanden. Obgleich die Iris durch die Verwachsung ibrer Wnzel abnorm weit vorgerickt wird, ist die vordere Kammer eher tief.

Die Iris ist schmal und atrophisch und zeigt ein ausgesprochenes $\mathrm{Ek}_{\text {- }}$ tropium des Pupillarrandes, an dem auch der Sphinkter Theil nimmt. Oben und oben innen, wo klinisch ein Irismangel bestand, fehlt die Iris nicht etwa, sondern bildet einen kurzen Stumpf, welcher den allerhöchsten Grad des Ektropiums zeigt, der Art, dass der Sphinkter vollkommen auf die Yorderfläche umgebogen ist und sein ursprünglich der Pupille zngekehrter Rand nunmehr bis in den Winkel reicht, wo die Hornhaut mit dem periphersten Theil der Iris verwachsen ist. $\mathrm{Da}$ also auf dieser Seite die Iris ganz in sich zusammengezogen ist, erscheint sie dicker als auf der gegenüberliegenden, ist aber doch in Folge der allgemeinen Atrophie auch hier noch eher schmal zu nennen. 
In Folge der Ektasie der Cornea liegt dieser Irisstumpf so peripher, dass er von vorn her durch den Conjunctivalansatz verdeckt und hierdurch klinisch unsichtbar wurde.

Bemerkenswerth ist, dass am Pigmentepithel der Iris Quellung und Wucherung der Zellen besteht, in derselben Art, wie es melrfach an den Augen von Diabetikern beobachtet wurde.

Die Ciliarfortsätze sind überall, am meisten aber oben innen nach einwärts gezerrt, der Ciliarmuskel wird von diesem Zuge gleichfalls betroffen und hat dadurch eine ganz ungewöhnliche Gestalt angenommen (vgl. Fig. 2).

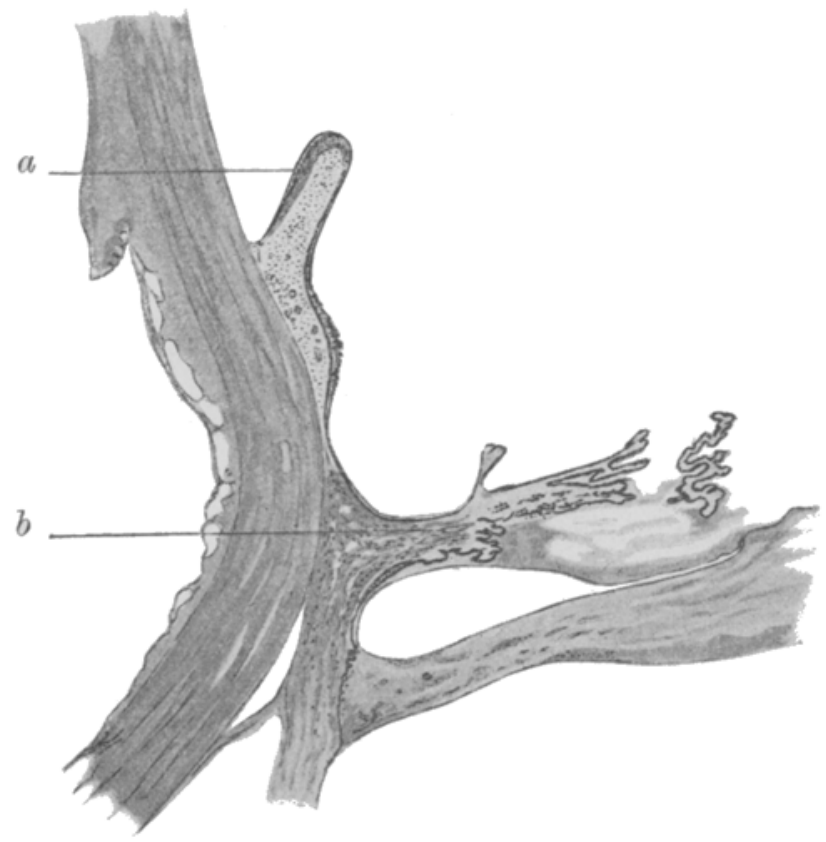

Fig. 2.

Die Ciliarfortsätze werden durch die Zonula nach innen gezogen, welche in fester Verbindung mit einer hinter der Linse gelegenen, vom Orbiculus ciliaris aus reichlich vascularisirten bindegewebigen Schwarte steht, die andererseits mit der abgelösten Retina verwachsen ist. In derselben findet sich reichlich hämatogenes Pigment sowie schlanchförmige Zellstränge, welche aus gewncherten, pigmentirten sowie unpigmentirten Epithelzellen des Ciliarkörpers bestehen.

Die Retina ist total atrophisch, sehr reichlich hämatogen pigmentirt und strangförmig abgelöst. Auch auf sie wirkt von innen her ein sehr starker Zug, was daran zu erkennen ist, dass der Opticus bis ins Innere des Bulbus hineingezerrt ist; ihn folgt hierbei die angrenzende Chorioidea. Der Sehnerv ist total atrophisch. - Hinter dem Corpus ciliare ist die Chorioidea eine Strecke weit von der Sklera abgehoben, da sich jedoch in 
diesem Raum in den Schnitten nur Celloidin findet, darf man wohl eine arteficielle Ablösung vermuthen.

Das Stroma der Chorioidea ist rollkommen pigmentios, die Membran ist besonders im hinteren Bulbusabschnitt aufgelockert, ziemlich kernarm, lässt aber an den Gefässen nichts sicher Pathologisches erkennen.

Der subretinale Raum ist oben innen von einer enormen Blutung eingenommen, die Blutkörperehen sind zusammengebacken und bilden vielfach ganz homogene Massen. Vorn dringen Bindegewebszïge jener oben erwähnten Schwarte in die Blntrng ein. Weniger reichliche Blutmassen finden sich temporal in vorderen Bulbusabsehnitt. Im Uebrigen ist der subretinale Raum von geronnenen Eiweissmassen erfüllt, solche sind anch in der vorderen und hinteren Kammer anzutreffen. Noch gut arhaltene rothe Blutkörperchen liegen in grosser Menge im vorteren Bulbusabschnitt der Innenfläche der Aderhaut auf.

Epikrise: Das wichtigste Ergebniss der vorliegenden Untersuchung ist, dass ein für die klinische Beobachtung rollständiger umschriebener Defect der Iris thatsächlich nicht durch ein Fehlen, sondern durch eine enorme Retraction derselben zu erklären ist, welch letztere durch ein totales Ektropium des Pupillarrandes entstanden ist. Dieses pflegt sich in glaukomatösen Augen zu entwickeln; anch in unserem Falle können wir einen Status glaucomatosus aus der Elitasie der Cornea, der Verwachsung der Irisperipherie mit derselben und dem Hartbleiben des Bulbus nach der Enucleation diagnosticiren.

Von grossem Interesse ist es, zu ermitteln, wann die ganze Erkrankung des Bulbus entstanden ist und welche von den Veränderungen wir als die primären anzusehen haben. Entweder ist das ganze Krankheitsbild als eine Verletzung des Augapfels durch Druck der Zange anzusehen, demnach ausgedehnte intraoculare Blutungen als primäre Veränderung zu betrachten, denen sich entzíndliche Vorgänge anschlossen, oder die Erkrankung war bereits intrauterin entstanden und bei der Geburt traten vielleicht spontan oder durch Zangendruck Blutungen hinzu, wie dies ja auch sonst bei Geburten, die mit oder olne Runsthilfe beendet werden, vorkommt.

Dass bei der Geburt grössere intraoculare Blutungen entstanden sind, ist vollkommen sicher, da ja am ersten Lebenstage nach der Beobachtung des Vaters die ganze Kammer voll Blut war. Die Angabe, dass drei Wochen post partum die Pupille "ganz verzerrt" und oben innen ein gelbrother Reflex aus der Tiefe zu sehen war, macht es wahrscheinlich, dass dex Befund an der Tris damals schon dem später von uns beobachteten glich, denn wenn die Tris oben innen nicht retrahirt gevesen wäre, so hätte man kaum die ziemlich peri- 
pher gelegene grosse Blutung so deutlich gesehen. Machen wir die Annahme, dass schon im Alter von drei Wochen der scheinbare Irisdefect vorhanden war, so werden wir dazu gedrängt, den Beginn der Augenerkrankung in die Fötalzeit zu verlegen; denn in drei Wochen kann sich wohl schwerlich ein Ektropium des Pupillarrandes bilden, das den Irisstumpf vollkommen hinter den Limbus verschwinden lässt. Wir hätten dann also eine schwere intrauterin entstandene Entzündung anzunelimen und die Blutungen wären ein mehr nebensächlicher Befund. Mit voller Sicherheit lässt sich diese Auffassung indessen nicht beweisen, da es nicht zweifellos ist, ob der. Irisdefect wirklich schon mit drei Wochen die gleiche Form hatte wie später.

Ebenso bleibt es unsicher, wodurch es zur Drucksteigerung und zur Ausbildung der fü Glankom mehr oder weniger charakteristischen anatomischen Veränderungen gekommen ist. Z weifellos ist nur, dass zur Zeit der Enucleation ein schwerer zur Atrophie führender Entzündungszustand im vorderen Uvealtractus bestand, mit Bildung einer cyclitischen Schwarte, welche durch Schrumpfung einen ausserordentlich starken Zug ausübte und zur Einwärtszerrung der Retina, der Ciliarfortsätze sowie der Bulbuswand an der Sklerocornealgrenze oben innen fuhrte. Bemerkenswerth ist dabei das Sichtbarwerden der Ciliarfortsätze im Pupillargebiet. In neuester Zeit sind einige klinisch beobachtete Fälle, in welchen diese Erscheinung vorlag, wegen ihrer Seltenheit mitgetheilt worden. Ich halte das Sichtbarwerden von Ciliarfortsätzen im Pupillargebiet nach 'Verletzungeu für nicht besonders selten und habe selber eine grössere Zahl solcher Fälle gesehen. Wichtig ist aber für mich, dass sowohl im Falle von Schweigger') wie in dem von Eversbusch ${ }^{2}$ ) das betreffende Auge etwas kleiner war als sein Partner und die Anamnese darauf hinweist, dass der Befund sehr wahrscheinlich seit Geburt bestand. Dort waren allerdings membranöse Staare vorhanden, deren Schrumpfung zur Verzerrung der Ciliarfortsätze führte, doch ist es kaum zu bezweifeln, dass es auch in meinem Falle zu Cataractbildung gekommen wäre. Im Schweigger'schen Falle bestanden auch Complicationen, denn nach' der Discission war kein rothes Licht zu erhalten. Ich halte es deshalb für wohl möglich, dass auch jene Fälle durch intrauterine Entzündung oder durch ein Geburtstrauma zu erklären sind.

In meinem Falle spricht noch gegen die Annahme, dass eine

$\left.{ }^{1}\right)$ Areh. f. Augenheilk. XLII. Bd. S. 171.

2) Münch. med. Wochenschr. 1901. Nr. 2. 
Verletzung dureh die Zange die Angenerkrankung erst herbeigefüht hat, das Fehlen von Excoriationen oder Blutungen an deu Lidern, sowie von Anzeichen für Knochenbrüche, die wohl kaum unbeachtet geblieben wären. In den mir aus der Literatur bekannt gewordenen Verletzungen des Auges durch die Zange haben solche Spuren des Tranmas in der Umgebung des Bulbus nicht gefehlt. Es ist auch nicht sehr wahrscheinlich, dass eine traumatische intraoculare Blutung ohne Perforation der Bulbuswand so schwere entzuindliche Veränderungen nach sich ziehen sollte. Da sich aber eine absolute Gewissheit nicht erlangen lässt, so verzichte ich auf eine weitere Erörterung und möchte nur darauf hinweisen, dass die Unsicherheit der Auffassung selbst in diesem Falle, der doch von Geburt an unter besonders interessirter äratlicher Beobachtung stand, zur Vorsicht malnt bei der Deutung mancher erst im späteren Lebensalter untersuchter Faille, deren Besonderheiten dazu führten, den Befund als angeborene Missbildung zu deuten und schon häufig Veranlassung zu recht gezwungenen Erklärungsversuchen gegeben haben, um ihn mit gewissen Phasen der Entwicklung des Augapfels in Zusammenhang zu bringen. So balte ich es z. B. für ganz wohl möglich, dass weisse Stränge und Faserhäute im Glaskörper, die im späteren Leben beobachtet werden, auf grössere wäbrend der Geburt entstandene Blutungen in den Glaskörperraum zurickzuführen sind.

Ich verzichte darauf zu erörtern, wie weit atypische Irisdefecte, die in der Literatur mitgetheilt sind, vielleicht in ähnlicher Weise wie in meinem Falle entstanden sein könnten, da ich zu bestimmteren Vorstellungen in dieser Hinsicht nicht babe gelangen können. Nur auf die Combination ron Buphthalmus mit angeborenem Irismangel möchte ich hinweisen und wenigstens die Möglichkeit betonen, dass hier der Irismangel analog meiner Beobachtung zu erklären wäre.

\section{Hochgradige einseitige Myopie und ausgedehnte markhaltige Nervenfasern bei einem $2^{1 / 2}$ Jahre alten Kinde.}

Das sonst ganz gesunde Kind, Maria Münch, wird in die Klinik gebracht, weil es zeitweise selielt. Familienanamnese negativ.

Leichter Strabismus converg. period. des rechten Anges, welches gleichzeitig etwas nach oben abweicht.

Rechts hochgradig myopische Refraetion. Noch aus $20 \mathrm{~cm}$ Entfernung bekomme ich (Myop 3 D) beim Hineinlenchten mit dem Spiegel ein vollkommen deutliches umgekehrtes Bild des Augenhintergrundes. Der Fundus ist äusserst pigmentarm, so dass die ganzen Chorioidealgefässe deutlich zu sehen sind, ganz im Gegensatz zum linken Auge, wo der Fundus gleich- 
mässig roth ist. Sehr ansgedelnte markhaltige Nervenfasem um die Papille, sonst keine Anomalie im Fundus.

Der vordere Bulbusabschnitt zeigt keine Prominenz, die Hornhäute sind an beiden Augen vollkommen gleich gross, ebenso die Tiefe der vorderen Kammer.

Linkes Auge ungefähr emmetropisch, in jeder Hinsicht normal.

Es dürfte selten die Gelegenheit gegeben sein, bereits in so fiuhem Alter das Bestehen sehr hochgradiger Myopie mit voller Sicherheit nachzuweisen. Dass hier nicht nur die Anlage zur Entwicklung von Myopie, sondern diese selbst angeboren war, dürfte kaum zu bezweifeln sein.

3. Finseitige parenchymatöse Hornhauttrübung, vordere Synechie ohne Hornhautperforation und doppelseitiges symmetrisches Aderhautcolobom nach oben aussen bei einem Neugeborenen.

Adolif Bullian, sechs Tage alt, wird am 22. XI. 1900 in die Augenklinik gebracht wegen eines weissen Fleckens an rechten Auge, der von der Hebamme und der Mutter am zweiten Lebenstage bemerkt worden war. Die Geburt hatte ziemlich lange gedauert, war aber ohne Kunsthilfe beendigt. Von vier Geschwistern eine Frühgeburt, eins früh gestorben. Ein fünf Jahre altes Kind hat, wie ich selber feststellen konnte, Strabismus convergens und Nystagmus rotatorius, ein anderes hat normale Augen, so weit man bei dem etwas ungeberdigen, zweijährigen Kinde feststellen kann.

Status praesens: R. A. Ganz leichte Excoriationen im äusseren Lidwinkel, im Bindelaautsack etwas Secret, Auge blass. Grosse, etwas nach unten aussen gelegene parenchymatöse Hornhauttrübung, Hornhautoberfläche an dieser Stelle matt. Kein Substanzverlust, Kammer flach. Auf Atropin tritt unten aussen eine vordere Synechie hervor, rom Pupillarrand spannt sich ein Faden nach der Hinterfäche des getrïbten Hornhautbezirks. Die Pupille erweitert sich im Ganzen nur unvollkommen auf Atropin. Fluorescein ergiebt eine sehr ausgesprochene tief liegende Grünfärbung in Gebiet der Homhanttribung.

26. XI. Befund regelmässig controlirt, ist unverändert. Täglich Atropin.

29. XI. Etwas mehr Secret im Conjunctivalsack; bei Druek auf den Thränensack entleert sich reichlich dicker Eiter aus den Thränenpunkten.

An der Tris fallt eine starke Hyperämie und grosse Resistenz der Pupille gegen Atropin auf. Fluorescein giebt noch ganz schwache Grünfürbung.

30. XI. Am Körper verdächtiges Exanthem, Excoriationen an den Fusssohlen und Abschuppungen an den Händen. Von der Kinderklinik wird mit Wahrscheinlichkeit hereditäre Lues angenommen. Deshalb wird eine Einreibungseur zu $0,5 \mathrm{~g}$ begonnen. Der Thränensack wird regelmässig ansgentrückt.

2. XII. Kein Secret mehr auszudrücken.

5. XII. Fluorescein giebt keine Färbung mehr. Hornhauttrübung wohl 
etwas aufgehell, man sieht wenigstens die vordere Synechie viel dentlicher als bisher.

22. XII. 20 Einreibungen gemacht. Es sind keine luetischen Elscheinungen mehr nachweisbar. Wegen Stomatitis und Gewichtsabnahme Cur ausgesetzt. Es wird taglich einmal Atropin gegeben.

4. I. 1901. Befund unverändert, nur zeigt sich im Centrum der Tribung anf Flnorescein wieder leichte Grinfärbung.

2:. I. 1901. Auge rollkommen blass, frei geöffinet. Hornhauttrubung vielleicht etwas lichter, in der Form aber unverändert. Oberfläche vollkommen glatt. Die vordere Synechie ist verschwunden, Pupille rund, aber dureh Atropin immer noch nicht vollig erweitert. Fluorescein giebt keine Spur von Firrbung.

Medien bis auf die Hornhauttribung klar. Nach oben aussen ron der normalen Papille findet sich auf beiden Augen in genau symmetrischer Lage und Grösse ein schräg ovaler, mehrere P. D. grosser, glänzend weisser, scharf begrenzter Bezirk, der besonders am nasalen Rande intensiv pigmentirt ist und keine $z$ weifellose Ektasie zeigt. Einzelne Netzhautgefässe ziehen darüber hinweg. Das Ausselen entspricht volkommen dem bei angeborenem Aderhautcolobom so oft beschriebenen. Wegen der Unruhe des Kindes gelang es mir nicht, eine Zeichnung aufzunehmen. Nirgends kronnte ich sonst im Augenhintergrunde eine Veränderung nachreisen.

Der Fall, welcher bezuglich der Combination dex vorgefundenen Anomalien soweit ich weiss ein Unicum darstellt, ist besonders interessant, weil es möglich war, durch die frühzeitige genane Lntersuchung die Natur der Hornhauttribung zu erkennen. Man braucht sich nur vorzustellen, dass das find, olne je behandelt zu. sein, in späterem Alter - sagen wir mit einem Jahre - zum ersten Mal in augenärztiche Beobachtung gekommen wäre. Die jetzt durch Atropin zum Reissen gebrachte vordere Synechie hätte dann noch bestanden in Verbindung mit einer der Anamnese nach sicher angeborenen Hornhauttrübung. Es ist kein Zweifel, dass man nun in dem gleichzeitigen Vorhandensein des doppelseitigen angeborenen Aderhautcoloboms den sichersten Beweis dafür erblickt hätte, dass auch die ibrigen Veränderungen als Hemmungsbildungen anzusehen seien! Thatsächlich handelte es sich aber um eine durch Erkrankung des Homhatendothels entstandene umschriebene parenchymatöse Trübung, wie die Grünfärbung durch Fluorescein nachwies. Wie diese Erkrankung zu Stande kam und wie lange vor der Geburt sie bereits vorhanden war, ist nicht zu entscheiden. Aypothetisch bleibt auch die Entstehungsweise der vorderen Synechie. Ich möchte es für wahrscheinlich halten, dass es bei einer lange dauernden, vielleicht gar bei jeder Geburt zu einer hochgradigen Abflachung der 
vorderen Kammer kommt, wobei eine Berührung zwischen Hornhaut und Iris stattfinden kann. An einer von Endothel entblössten Stelle kann dann leicht eine Verklebung eintreten. Auf dieselbe Weise wie in diesem Falle können Verwachsungen der persistirenden Pupillarmembran mit der Hornhaut erklärt werden, wenn keine Perforation der Hornhaut vorangegangen war. Ausser den von mir in GraefeSämisch's Handbuch, S. 62 angeführten Fällen liegt noch eine nene Beobachtung von Schapringer (New Yorker Med. Monatsschr. Bd. XII, Nr. II) ror.

Symmetrische Aderhautcolobome nach oben aussen an beiden Augen sind bisher überhaupt noch nicht beobachtet worden. Für die Klarstellung ihrer Genese liefert der Fall keinen Beitrag. Wenigstens gehe ich nicht so weit, aus den sonstigen hier beobachteten pathologischen Veränderungen den Schluss zu ziehen, dass diese Colobome Entzündungsproducte sind. Hierzu berechtigt auch noch nicht die Möglichkeit, dass hereditäre Lues vorlag. Ich bemerke noch, dass die Collegen von der Kinderklinik nach genauer Ausforschung und Untersuchung des Vaters die Diagnose der Lues für sehr zweifelhaft hielten.

Herrn Professor Leber danke ich bestens für die freundliche Ueberlassung der interessanten Beobachtungen.

\section{Erklärung der Abbildungen.}

Fig. 1. Uebersichtsoild von Fall $\mathrm{T}$.

a Irisstumpf an der Stelle des scheinbaren Irismangels.

b Iriswurzel mit der Hornhaut verwachsen.

$c$ Einwärts gezerrte Ciliarfortsätze.

d Bindegewebsschwarte und vorderster Theil der abgelösten und degenerirten Netzhaut der grösste Theil der Netzhaut befindet sich in anderen Schnittebenenj.

e Blutung.

Fig. 2. a Irisstumpf mit totalem Elstropium des Pupillarrandes, einschliesslich des Sphinkters.

$b$ Einwärtszerrung des Ciliarmuskels, der dadurch eine ganz veränderte Gestalt gewonnen hat. 